EPJ Web of Conferences 64, 04006 (2014)

DOI: $10.1051 /$ epjconf/ 20146404006

(C) Owned by the authors, published by EDP Sciences, 2014

\title{
Interactions between exoplanets and the winds of young stars
}

\author{
A. A. Vidotto ${ }^{1, a}$, M. Opher ${ }^{2}$, V. Jatenco-Pereira ${ }^{3}$, and T. I. Gombosi ${ }^{4}$ \\ ${ }^{1}$ SUPA, School of Physics \& Astronomy, University of St Andrews, North Haugh, St Andrews, KY16 9SS, UK \\ ${ }^{2}$ Boston University, 725 Commonwealth Ave, Boston, MA, 02215, USA \\ ${ }^{3}$ Universidade de São Paulo, Rua do Matão 1226, São Paulo, SP 05508-090, Brazil \\ ${ }^{4}$ University of Michigan, 1517 Space Research Building, Ann Arbor, MI, 48109-2143, USA
}

\begin{abstract}
The topology of the magnetic field of young stars is important not only for the investigation of magnetospheric accretion, but also responsible in shaping the large-scale structure of stellar winds, which are crucial for regulating the rotation evolution of stars. Because winds of young stars are believed to have enhanced mass-loss rates compared to those of cool, main-sequence stars, the interaction of winds with newborn exoplanets might affect the early evolution of planetary systems. This interaction can also give rise to observational signatures which could be used as a way to detect young planets, while simultaneously probing for the presence of their still elusive magnetic fields. Here, we investigate the interaction between winds of young stars and hypothetical planets. For that, we model the stellar winds by means of 3D numerical magnetohydrodynamic simulations. Although these models adopt simplified topologies of the stellar magnetic field (dipolar fields that are misaligned with the rotation axis of the star), we show that asymmetric field topologies can lead to an enhancement of the stellar wind power, resulting not only in an enhancement of angular momentum losses, but also intensifying and rotationally modulating the wind interactions with exoplanets.
\end{abstract}

\section{Three-dimensional numerical simulations of stellar winds}

The magnetic fields of young stars play fundamental roles in the Physics of accretion. Their topologies are, however, not only important for the investigation of magnetospheric accretion, but also responsible for shaping the large-scale structure of stellar winds, which are crucial for regulating the rotation evolution of stars. In [1-6], we modelled stellar winds by means of three-dimensional (3D) magnetohydrodynamics (MHD) simulations. In particular, in $[2,3]$ we investigate the winds of young stars and how they can interact with newborn planets. We adopte simplified topologies of the stellar magnetic field. To simulate the stellar winds, we use the 3D MHD numerical code BATS-R-US [7], which solves the coupled system of ideal MHD equations for the continuity of mass and momentum, conservation of energy and magnetic field induction. Figure 1a shows the magnetic field configuration adopted in the simulations at initial instant $t_{0}$. The dipolar magnetic moment $\mathbf{m}$ is tilted with respect to the stellar rotation axis $\Omega$ by an angle $\theta_{t}$. The stellar wind particles and magnetic field lines interact with each other self-consistently. We assume a typical young star with mass $0.8 M_{\odot}$, radius $R_{\star}=2 R_{\odot}$ and rotation period of 1 day. The magnetic field intensity at the magnetic poles of the star

\footnotetext{
a e-mail: Aline.Vidotto@st-andrews.ac.uk
} 

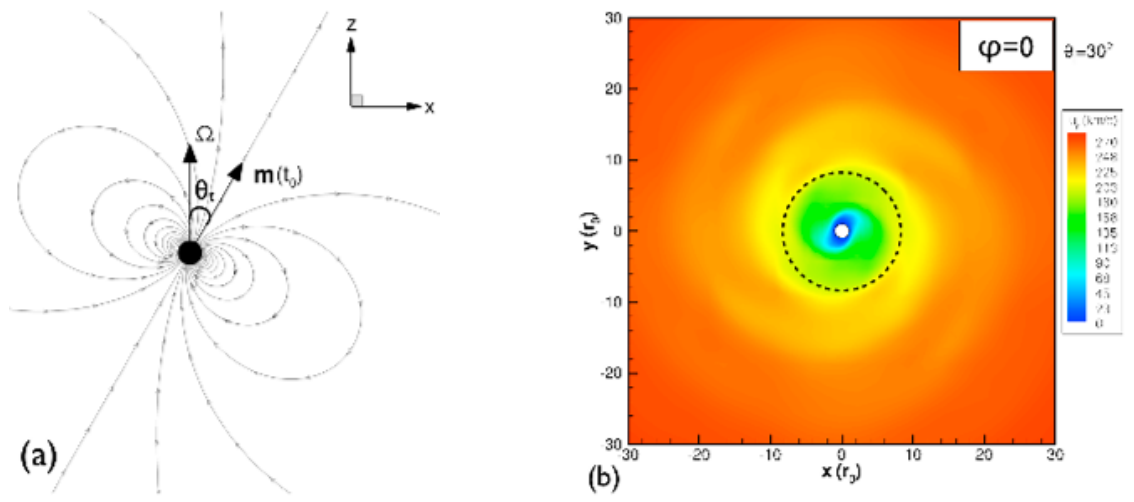

Figure 1. (a) Magnetic field configuration adopted in the simulations at initial instant $t_{0}$. The dipolar magnetic moment $\mathbf{m}$ is tilted with respect to the stellar rotation axis $\Omega$ by an angle $\theta_{t}$. Adapted from [3]. (b) A hypothetical hot-Jupiter orbiting in the equatorial plane $x y$ of a young star would interact with a stellar wind with varying conditions (e.g., velocities) along its orbit (illustrated by a dashed circle).

is $B_{0}=1 \mathrm{kG}$, the temperature and density at the base of the wind are $T_{0}=10^{6} \mathrm{~K}$ and $10^{-11} \mathrm{~g} \mathrm{~cm}^{-3}$, respectively. Figure 2 shows the evolution of the stellar wind velocity and magnetic field lines at different stellar phases of rotation $\varphi$. Note that the wind is modulated with the same period of the star. Figure $1 \mathrm{~b}$ shows the velocity profile of the stellar wind at the rotational equatorial plane, for the case where $\theta_{t}=30^{\circ}$. Note that the symmetry about the $z$-axis on the wind properties is lost in the case of a tilted dipolar magnetic field.

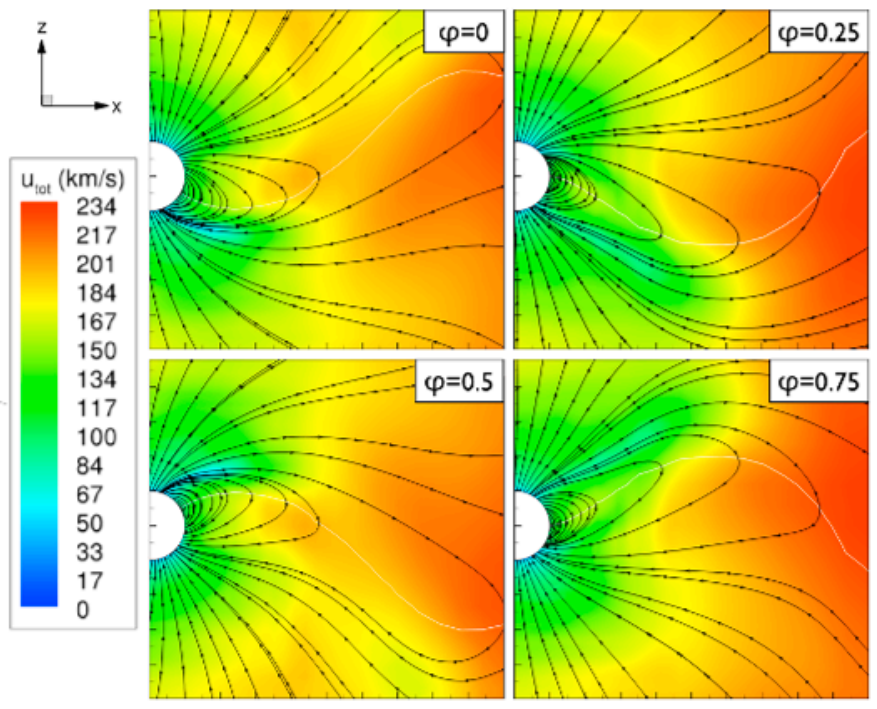

Figure 2. Evolution of the stellar wind velocity and magnetic field lines at different stellar phases of rotation $\varphi$. The wind is modulated with the same period of the star. Adapted from [3]. 


\section{Stellar wind interaction with planets}

Because stellar winds of young stars are believed to have enhanced mass-loss rates compared to those of cool MS stars, the interaction of winds with newborn exoplanets might affect the early evolution of exoplanetary systems. Figure $1 \mathrm{~b}$ illustrates the orbital path of a hypothetical hot-Jupiter (dashed circle) orbiting in the equatorial plane of a young star. Because the wind characteristics vary along the planetary orbit due to the asymmetry of the stellar magnetic field, an exoplanet orbiting in the equatorial plane of a young star would interact with a stellar wind with varying conditions along its orbit. As the stellar wind impacts on a magnetised planet, it compresses its magnetosphere. As a result, the magnetospheric size $r_{M}$ varies along the planetary orbit. Pressure balance between the wind total pressure and the planet total pressure requires that, at a the magnetopause distance $r_{M}$ from the planet, $p_{\text {tot }}=B_{p, r_{M}}^{2} / 8 \pi+p_{p}$, where $p_{\text {tot }}$ is the stellar wind total pressure (i.e., the sum of thermal, magnetic and ram pressures), $B_{p, r_{M}}$ the planetary magnetic field intensity at a distance $r_{M}$ from the planet centre and $p_{p}$ is its thermal pressure. We neglect the thermal pressure of the planet. Assuming the planetary magnetic field is dipolar, we have that $B_{p, r_{M}}=B_{p \text {,eq }}\left(R_{p} / r_{M}\right)^{3}$, where $R_{p}$ is the planetary radius and $B_{p \text {,eq }}$ its surface magnetic field at the equator. For a planetary dipolar axis aligned with the rotation axis of the star, the magnetospheric size of the planet is given by

$$
\frac{r_{M}}{R_{p}}=\left[\frac{B_{p, \mathrm{eq}}^{2}}{8 \pi p_{\mathrm{tot}}}\right]^{1 / 6}
$$

Figure 3a shows how the sizes of planetary magnetospheres vary as a function of the stellar phase of rotation. For illustration purposes, we take a planet located at $\{x, y, z\}=\{5,0,0\} R_{\star}$, which is equivalent as an orbital radius of $\approx 0.05$ au. Because of our artificial selected orbital position, the lines presented in Figure $3 \mathrm{a}$ are merely symbol connectors and do not represent actual variation of $r_{M}$. The hypothetical planet is assumed to have an intrinsic dipolar field of $50 \mathrm{G}$. Note that the larger $\theta_{t}$ is, the largest influence the wind will have on the size of the planet's magnetosphere.

The interaction of a magnetised wind with a magnetised exoplanet can give rise to observable signatures which could be used as a way to detect young planets [8-10], while simultaneously probing the presence of their still elusive magnetic fields [5, 8]. Powered by the stellar wind, magnetic reconnection generate electrons that propagate along the planet's magnetic field lines and produce electron cyclotron radiation at radio wavelengths. The power released in the interaction depends on the planet's magnetic field intensity, its orbital radius, and on the stellar wind local characteristics [5]. Figure $3 \mathrm{~b}$ shows the predicted planetary radio emission generated in the interaction between a hot-Jupiter and the stellar wind. The shaded area lies between maximum and minimum powers that can be released in the interaction, according to the rotational phase of the star. The red curve assumes an aligned stellar dipole. For comparison, Jupiter radio emission is $\approx 10^{10.5} \mathrm{~W}$. We find that a close-in Jupiter-like planet orbiting at 0.05 au presents a radio power that is $\sim 5$ orders of magnitude larger than the one observed in Jupiter, which suggests that the stellar wind from a young star has the potential to generate strong planetary radio emission that could be detected with LOFAR. This radio power is modulated by the rotation of the star and the orbital motion of the planet.

It has been suggested that torques from the stellar wind can remove orbital angular momentum of the planet, causing planetary migration [11]. Using the results of our simulations, we analyse whether winds from misaligned stellar magnetospheres could cause a significant effect on planetary migration. Figure $3 \mathrm{c}$ shows the timescales $\tau_{w}$ for planetary migration caused by torques from the stellar wind. The solid line assumes a purely aligned (axisymmetric) dipolar field and the dashed line assumes an oblique dipole with $\theta_{t}=30^{\circ}$. Compared to the aligned case, we show that the time-scale $\tau_{w}$ for an appreciable radial motion of the planet is shorter for larger misalignment angles. While for the aligned 


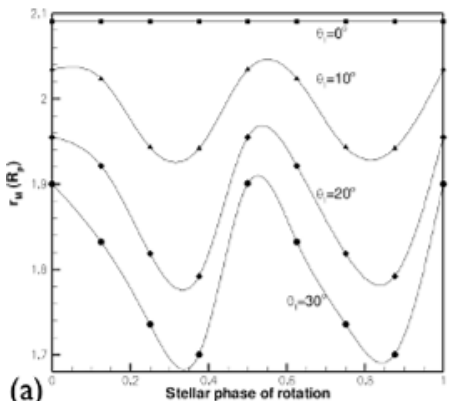

(a)

Stellar phase of rotation

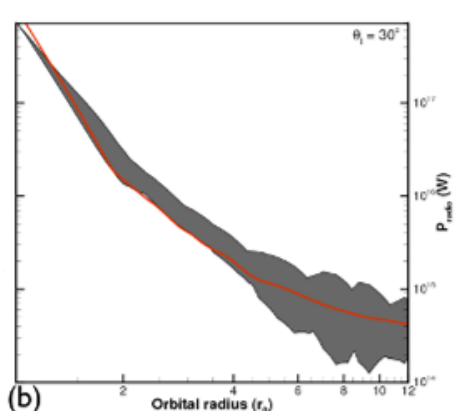

(b)

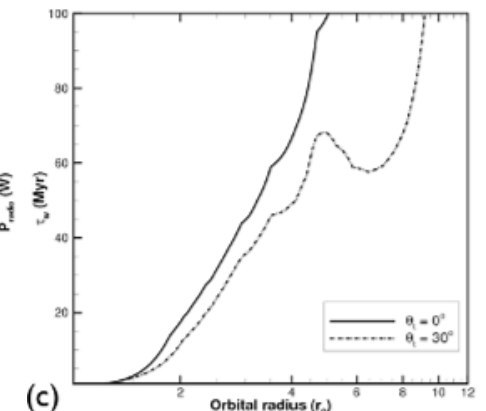

Figure 3. (a) Sizes of planetary magnetospheres of a hypothetical planet orbiting at 0.05 au, with an intrinsic dipolar field of 50 G. (b) Predicted planetary radio emission generated in the interaction between a hot-Jupiter and the stellar wind. The shaded area lies between maximum and minimum powers that can be released in the interaction, according to the rotational phase of the star and position of the planet. The red curve assumes an aligned stellar dipole. (c) Timescales for planetary migration caused by torques from the stellar wind. Complex stellar fields are more efficient in causing planetary migration than aligned fields. Adapted from [3].

case $\tau_{w} \approx 100 \mathrm{Myr}$, for a stellar magnetosphere tilted by $\theta_{t}=30^{\circ}, \tau_{w}$ ranges from $\sim 40$ to $70 \mathrm{Myr}$ for a planet located at a radius of 0.05 au. This indicates that complex stellar fields are more efficient in causing planetary migration than aligned fields. Further reduction on $\tau_{w}$ might occur for even larger misalignment angles and/or different wind parameters.

\section{Concluding Remark}

Stellar winds carry angular momentum of the star. In the simulations presented in [3], angular momentum losses are enhanced by a factor of 2 as one goes from the aligned case to the case where the dipole is tilted by $90^{\circ}$. In addition to an enhancement of angular momentum losses, we showed that asymmetric field topologies can intensify and rotationally modulate the wind interactions with exoplanets.

\section{References}

[1] A.A. Vidotto, M. Opher, V. Jatenco-Pereira, T.I. Gombosi, ApJ 699, 441 (2009)

[2] A.A. Vidotto, M. Opher, V. Jatenco-Pereira, T.I. Gombosi, ApJ 703, 1734 (2009)

[3] A.A. Vidotto, M. Opher, V. Jatenco-Pereira, T.I. Gombosi, ApJ 720, 1262 (2010)

[4] A.A. Vidotto, M. Jardine, M. Opher, J.F. Donati, T.I. Gombosi, MNRAS 412, 351 (2011)

[5] A.A. Vidotto, R. Fares, M. Jardine, J.F. Donati, M. Opher, C. Moutou, C. Catala, T.I. Gombosi, MNRAS 423, 3285 (2012)

[6] M. Jardine, A.A. Vidotto, A. van Ballegooijen, J.F. Donati, J. Morin, R. Fares, T.I. Gombosi, MNRAS 431, 528 (2013)

[7] K.G. Powell, P.L. Roe, T.J. Linde, T.I. Gombosi, D.L. de Zeeuw, J. Chem. Phys. 154, 284 (1999)

[8] A.A. Vidotto, M. Jardine, C. Helling, ApJ1 722, L168 (2010)

[9] A.A. Vidotto, M. Jardine, C. Helling, MNRASl 411, L46 (2011)

[10] A.A. Vidotto, M. Jardine, C. Helling, MNRAS 414, 1573 (2011)

[11] R.V.E. Lovelace, M.M. Romanova, A.W. Barnard, MNRAS 389, 1233 (2008) 